\title{
Real-time Short Message Services Application to Reduce Operating Room Turnover Time
}

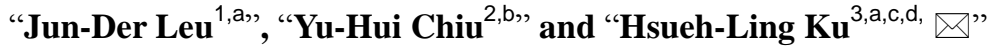 \\ ${ }^{a}$ National Central University, Tw,R.O.C. \\ ${ }^{b}$ Wan Fang Hospital, Taipei Medical University,Tw, R.O.C. \\ C Cathay General Hospital, Tw, R.O.C. \\ d Ching Kuo Institute of Management and Health,Tw, R.O.C. \\ $\bowtie$ E-mail: 201385@cgh.org.tw
}

\begin{abstract}
This study examined the interaction between computerized operating room scheduling systems and operators. The study evaluated the management performance of operating room scheduling in terms of the turnaround time. Operating rooms deliver instant medical information regarding surgical patients to operators through automated short message services (SMS), combined with a computerized scheduling system; This study assessed the difference in operational efficiency before and after the function was integrated. The turnover time reduced from 25.20 minutes to 24.89 minutes, with the standard deviation of 19.85 in the control group and 16.64 minutes in the experimental group. The $\mathrm{P}$ value was 0.000 while the $\mathrm{t}$ value was 4.889 , suggesting a statistical difference. The telephone costs of operating rooms were reduced from NTD 6 per operation to NTD1.5; the satisfaction rate was $96.1 \%$ among operators and 96.6 $\%$ among operating room nurses. Operating rooms' application of automated SMS by computerized scheduling systems has shown the advantages of being economic, efficient, instant, convenient, and accessible at all times. In the meantime, it establishes a platform for communication between the two parties. The application not only properly controls the turnover time in each operating room, but also enhances the efficiency of operating room scheduling, reduces telephones costs of operating rooms, and improves the satisfaction of medical personnel with their work.
\end{abstract}

Keywords: Computerized Scheduling System, Automated SMS, Turnover Time. 


\section{Introduction}

The main reasons causing the dissatisfying management performance of operating room scheduling include hindered schedules of operating rooms, underuse, or overuse of operating rooms, prolonged turnover time, or increased non-operative time. Accordingly, whether or not the schedules of each operating room of the day are appropriately managed can be indicated by if the turnover time between two operations is reasonable, to evaluate the management performance of operating rooms $[2,6,5]$.

Open communication between medical team personnel is essential to constructing a safe environment of medical healthcare for surgical patients; moreover, it is an indicator for medical institutes to evaluate the operation performance of the units. Communication between medical team personnel is vital; Sixty-six percent of all the sentinel events fundamentally result from improper communication. Information delivered during communications should be intact, clear, precise, and instantaneous to build an effective bridge that serves the function of good communication [ 5$]$. Operators are like band directors or steerers, who dominate the treatment processes for surgical patients. After operators enter the basic information of the scheduled patients (sex, age, diagnoses, methods of anesthesia, and names of operations), equipment for operations and anticipated operation time into the computerized scheduling system on the day before the operations, the in-charge personnel on the day of the operations should contact the operators momentarily to confirm the scheduled time of operations. In the meantime, the operators usually wait for notices from operating rooms in offices, so that they can leave for operating rooms on time (as scheduled) and proceed to operations on patients with other medical team personnel [3] .

Cell phone and PDA users use calendars, learning timetables and electronic notes to replace traditional paper notebooks and other calendars to manage their daily schedules, appointments, and work progress. These mobile devices can be used at all times; furthermore, the features of automatic reminders of upcoming critical information and events and the repeated accessibility allow users not to miss or forget any important events [ 1 ] . In-charge personnel in operating rooms provide a decision-making mechanism for the computerized scheduling system, which should 
include an automated decision-making system and necessary information for relevant personnel, to reduce the time waiting for patients and surgeons. As well as the non-operative time caused by the prolonged turnover (and overuse time due to congested schedules), the management performance of operating rooms is improved [4]..As a result, it is important how operating rooms: apply computerized scheduling systems; combine automated SMS on medical information in delivering momentary information about patients' conditions so that operators can receive detailed instant medical information about the surgical patients in time; plan working procedures in advance; arrive in the operating rooms on time; respond and initiate various operation-related medical activities for patients; and enable the whole crew to participate in the operations successfully.

\section{Research Method}

2.1. Research Map: With the application of the computerized scheduling system, patients' instant medical information is delivered to operators through automated SMS as soon as the patients arrive in operating rooms. (Figure 1) .

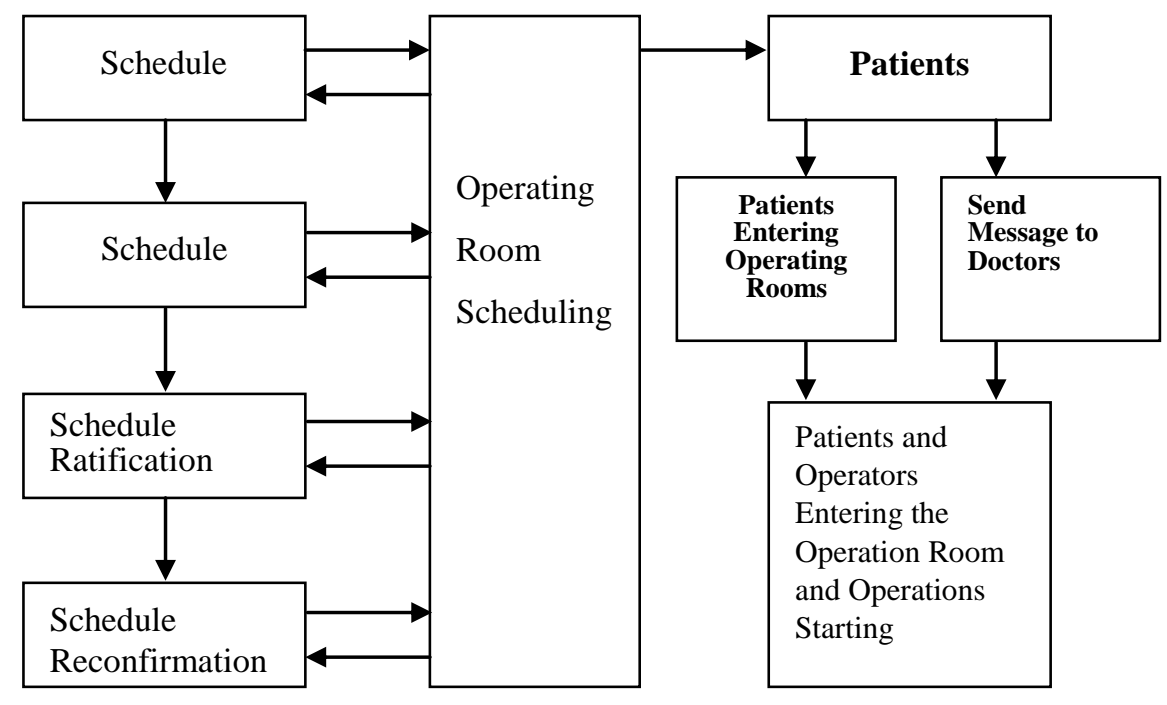

Figure 1. Computerized Scheduling System

2.2. Research Period: Operations performed every day from 0800 to 1700 in the ten operating rooms numbered $1,2,3,4,6,7,8,9,10$ and 11 from July $7^{\text {th }}$ to July $19^{\text {th }}$ 
in 2008 were collected into the control group (before the integration), while operations performed from January $5^{\text {th }}$ to January $17^{\text {th }}$ in 2009 were collected into the experimental group (after the integration).

2.3. Research Subjects: The basic information of surgical patients in the operating rooms of a medical center in Taipei City (a. medical information of patients included patients' names, case numbers, ages, bed numbers, diagnoses, names of operations, operators, and check-in times; b. patient-related information included arrival time in operating rooms, exit time from operating rooms, arrival time of the second operation, exit time, operators, and operating room nurses).

2.4. Definition: Turnover time refers to the time from patient-out to next patient-in between two operations.

2.5. Hospital Profile: The case study was conducted at a medical center in Taipei City, Taiwan in which more than $99 \%$ of the patients are covered by the National Health Insurance. There are a total of 750 beds in the hospital and 14 operating rooms, which provide 24-hour services. Approximately 23,000 operations are performed each year and dispersed in 12 sub-specialties, including general surgery, neurosurgery, plastic surgery, orthopedics, urology, obstetrics and gynecology, ophthalmology, otolaryngology, cardiac surgery, rectum surgery, dental surgery, and thoracic surgery. The priority of using operating rooms was based on the numbers of operations in past years of each department, which were transformed into scores. With regards to computerizing the operating room scheduling system, the computerized system has been applied since 2005, which allows operators to enter patients' information into the scheduling system through the internet at any corner in the hospital on the day before operations. After the in-charge personnel on the day of operations confirm the information based on the operating room protocols of the departments, the operations can be performed on time.

\section{Results and Discussion}

\subsection{Evaluation of Turnover Time}

3.1.1 Data Analysis on Patients' Anesthesia of the Second Operation: There were 410 
surgical patients collected into the control group, with $60.5 \%$ of them having general anesthesia, $11.6 \%$ having epidural anesthesia and $27.9 \%$ local anesthesia. A total of 379 surgical patients were collected into the experimental group, with $56.2 \%$ of them having general anesthesia, $9.5 \%$ having epidural anesthesia and $34.3 \%$ local anesthesia. Detailed information is presented in Appendix Table 1.

Table 1: Data Analysis on Patients' Anesthesia of the Second Operation

\begin{tabular}{|c|c|c|c|c|}
\hline & Control & Control & Experimental & Experimental \\
\hline Method of & Group & Group & Group & Group \\
\hline \multirow[t]{2}{*}{ Anesthesia } & Number of & Percentage & Number of & Percentage \\
\hline & Case & $(\%)$ & Case & $(\%)$ \\
\hline \multicolumn{5}{|l|}{ General } \\
\hline \multicolumn{5}{|l|}{ Anesthesia } \\
\hline \multicolumn{5}{|l|}{ Epidural } \\
\hline \multicolumn{5}{|l|}{ Anesthesia } \\
\hline \multicolumn{5}{|l|}{ Local } \\
\hline & 114 & $27.9 \%$ & 130 & $34.3 \%$ \\
\hline \multicolumn{5}{|l|}{ Anesthesia } \\
\hline Total & 410 & $100 \%$ & 379 & $100 \%$ \\
\hline
\end{tabular}

3.1.2 Data Analysis on Turnover Time : The maximum value in the control group was 140 minutes and the minimum value was 5 minutes; the average was 25.20 minutes and the standard deviation was 19.85 minutes. The maximum value in the experimental group was 130 minutes and the minimum value was 5 minutes; the mean was 24.89 minutes and the standard deviation was 16.64 minutes. The $\mathrm{P}$ value from the comparison between the two groups was 0.000 and $t$ value was 4.889 . Detailed information is presented in Appendix Table 2. 
Table 2: Data Analysis on Turnover Time

Turnover Time

Items

$$
\text { Maximum Minimum Mean SD }
$$

Control 140 5 25.20 19.85

group 40

Experimental

Group

\subsection{Evaluation of Cost-Benefit}

In Taiwan, the average fee for cell phone calls is NTD 6 per phone call ( 1 minute); the average fee for SMS between cell phones (outside the network) is NTD 2.5 while it is NTD1.5 between computers and cell phones (inside the network). During the four weeks of research period in this study, 1,025 pieces of short messages had been sent from computers to cell phones, with a total charge of NTD 1,537.5. Conversely, if 1,025 calls were made and charged by the average rate, the total charge would have been NTD 6,150; this indicates that $75 \%$ of the expense was saved. Assuming that 23,000 people in the targeted hospital receive surgeries in a year, the expense saved should be $(6 \times 23,000)-(1.5 \times 23,000)=$ NTD138,000 - NTD34,500 =NTD103,500 .

\subsection{Evaluation of Satisfaction}

In total, 51 operators were inquired, and among all, $98 \%$ of them were satisfied that the automated short messages about patients' medical information contribute to a smoother scheduling of medical work.In total, 58 Operating Room Nurses were inquired, and among all, $94.8 \%$ of them emphasized the increase of workload when having to contact operators by cell phones (control group) at patients' arrival in the operating rooms. 


\section{Conclusion}

The automated SMS notification and reminder on surgical patients' medical information in this study serves the function of: 1) reducing turnover time; 2) decreasing cell phone fee of operating rooms;3) increasing operators' satisfaction; and 4) increasing operating room nurses' satisfaction. Applying the system properly to the service process can help personnel understand patients' most current conditions in time and thus, contribute to the satisfying results of necessary treatments. However, medical quality should be improved constantly to achieve perfection. The progressed computerized informational system should be fully utilized in the future for encouraging medical personnel to increase communication, reduce mistakes, and assist in decision-making to ensure the safety of every patient and to achieve the purposes of treatments.

\section{Reference}

(1) Corlett, D., Sharples, M., Bull, S., \& Chan, T. Evaluation of a mobile learning organiser for university students. Journal of Computer Assisted Learning.2005; 21(3): 162-170.

(2) Dexter, F., Macario, A., Changing Allocations of Operating Room Time From a System Based on Historical Utilization to One Where the Aim is to Schedule as Many Surgical Cases as Possible.2002. Anesthesia \& Analgesia; 94(5):1272-9.

〔3] Dexter F., Traub R D., \& Macario A. How To Release Allocated Operating Room Time To Increase Efficiency: Predicting Which Surgical Service Will Have The Most Underutilized Operating Room Time. Anesthesia and analgesia. 2003; 96(2):507-12.

〔4〕 Dexter EU., Dexter F., Masursky D., Garver MP., Nussmeier NA.Both bias and lack of knowledge influence organizational focus on first case of the day starts. Anesth $2009 ; 108(4): 1257-61$.

〔5 J JCAHO.Root Causes and Percentages for Sentinel Events (All Categories) January1995-December 2005

〔6〕 McIntosh, Catherine M, F., Dexter, F., Epstein, Richard H. The Impact of Service-Specific Staffing, Case Scheduling, Turnovers, and First-Case Starts on Anesthesia Group and Operating 
Room Productivity: A Tutorial Using Data from an Australian Hospital. Anesthesia \& Analgesia.2006; 103(6):1499-1516. 